\title{
DOI: 10.33947/1981-741X-v20n2-4835 \\ DE QUEM É O VERDE URBANO? UMA ANÁLISE GEOTECNOLÓGICA DA INIQUIDADE ARBÓREA DE SÃO CARLOS, SP
}

\section{WHOSE IS THE URBAN GREEN? A GEOTECHNOLOGICAL ANALYSIS OF ARBOREAL INIQUALITY IN THE MUNICIPALITY OF SÃO CARLOS, SP}

João Vitor Roque Guerrero', Daniel Tonelli Caiche², Rubia Gomes Morato³, Fernando Shinji Kawakubo4, Guilherme Augusto Verola Mataveli 5

Submetido em: 20/11/2021

Aprovado em: 03/12/2021

\section{RESUMO}

As áreas arbóreas prestam serviços ecossistêmicos essenciais aos seres humanos, principalmente em ambientes urbanos como forma de resiliência da natureza perante a intervenções antrópicas de grande impacto. Entretanto, o acesso a esse recurso nem sempre é igualitário dentre todas as camadas da sociedade, gerando iniquidades arbóreas ao longo dos municípios. Nesse sentido, o objetivo deste estudo foi utilizar técnicas de sensoriamento remoto, geoprocessamento e análise de dados geográficos para analisar a iniquidade arbórea do município de São Carlos, SP. Os resultados demonstraram que o perímetro urbano de São Carlos contém $11 \%$ de cobertura arbórea que, entretanto, ainda não é acessível a todos os cidadãos, principalmente aquelas áreas que estão segregadas pelos portões de condomínios fechados de alto padrão e àquelas preservadas pelas universidades locais. Ainda assim, foram identificadas áreas de remanescentes florestais em áreas periurbanas que prestam serviços ecossistêmicos essenciais em áreas públicas, que merecem atenção para que possam ser utilizadas como serviços culturais aos cidadãos locais.

PALAVRAS-CHAVE: Iniquidade Arbórea. SIG. CBERS 4A. Desenvolvimento Sustentável.

\section{ABSTRACT}

Tree areas provide essential ecosystem services to human beings, especially in urban environments as a form of nature's resilience to high-impact anthropic interventions. However, access to this resource is not always equal among all strata of society, generating arboreal iniquities across municipalities. In this sense, the objective of this study was to use remote sensing techniques, geoprocessing and geographic data analysis to analyze the arboreal iniquity in the city of São Carlos, SP. The results showed that the urban perimeter of São Carlos contains $11 \%$ of tree cover which, however, is still not accessible to all citizens, especially those areas that are segregated by the gates of high-end gated condominiums and those preserved by local universities. Even so, areas of forest remnants in periurban areas were identified that provide essential ecosystem services in public areas, which deserve attention so that they can be used as cultural services to local citizens.

KEYWORDS: Arboreal Iniquity. GIS. CBERS 4A. Sustainable Development.

\footnotetext{
1 Ph.D. em Ciências Ambientais. Universidade Federal de São Carlos

2 Ph.D. em Ciências Ambientais. Prefeitura Municipal de São Carlos

3 Ph.D. em Geografia Humana. Universidade de São Paulo

4 Ph.D. em Geografia Física. Universidade de São Paulo

5 Ph.D. em Geografia Física. Instituto Nacional de Pesquisas Espaciais
} 


\section{INTRODUÇÃO}

Com o contínuo processo de expansão dos limites e das populações urbanas, a natureza remanescente dentro das cidades torna-se cada vez mais importante, devido à promoção de diversos benefícios que melhoram a experiência de vida nas cidades (ZHAO et al., 2013). Esses benefícios podem ser relacionados a aspectos ecológicos como captura de contaminantes transportados pelo ar e pela água (KUEHLER; HATHAWAY; TIRPAK, 2017), regulação do microclima urbano e atenuação do efeito de ilha de calor (LIVESLEY; MCPHERSON; CALFAPIETRA, 2016; WERBIN et al., 2020), aumento da infiltração e retenção de águas pluviais mitigando os problemas de enchentes (ELY; PITMAN, 2013; SHACKLETON et al., 2015) e alagamentos entre outros (KIMPTON, 2017).

Outros benefícios que têm ganhado destaque nas mídias e em revistas científicas estão relacionados aos efeitos positivos na saúde humana, como aumento da resposta imunológica (TZOULAS et al., 2007), melhorando a eficácia do exercício (ULMER et al., 2016) e reduzindo ansiedade (TZOULAS et al., 2007), depressão (RUGEL et al., 2019), níveis de cortisol (WARD THOMPSON et al., 2012) e pressão arterial (DAVERN et al., 2017), além de aumentar a capacidade de aprendizagem e laboral das pessoas (KIMPTON, 2017; MCCORMICK, 2017).

Benefícios relacionados à vida social também tem sido objetos de estudo e mais uma vez indicam que a presença das árvores nas cidades influencia positivamente na vida das pessoas como fomentar o apego ao lugar (WATKINS et al., 2018) e confiança entre grupos (TYRVÄINEN et al., 2014); e reduzir a violência social (DONOVAN; PRESTEMON, 2012; GILSTAD-HAYDEN et al., 2015; KIMPTON, 2017).

Apesar dos já conhecidos benefícios das árvores nas cidades, observa-se um corpo crescente de pesquisas que demonstram a existência de um padrão em relação à distribuição de árvores nos territórios urbanos. Uma parte da comunidade de pesquisadores que se dedicam à temática da Floresta Urbana, têm levantado uma série de preocupações relacionadas às desigualdades de distribuição e acesso a áreas arborizadas, a chamada Iniquidade Arbórea, uma vez que determinados grupos sociais e comunidades não usufruem igualmente no uso dessas áreas. Cidades ao redor do mundo, nos diferentes continentes, apresentam uma disparidade na distribuição das áreas arborizadas nos centros urbanos (SCHWARZ et al., 2015; YOU, 2016; FERGUSON et al., 2018; NESBITT et al., 2019; LIOTTA et al., 2020).

É crescente o volume de análises sobre como as áreas arborizadas estão distribuídas no território e as barreiras que dificultam o acesso a elas. Alguns estudos compararam a quantidade de áreas verdes entre diferentes grupos sociais, utilizando como variáveis de análise o índice internacional de desigualdade - GINI, a origem étnica da população e ainda o nível de escolaridade e padrão construtivo das residências (MCCORMICK, 2017; FERGUSON et al., 2018)

Apesar de cada realidade ser construída de diversos aspectos e singularidades, os trabalhos têm indicado uma tendência: que as áreas arborizadas são menos presentes e acessíveis em 
DOI: $10.33947 / 1981-741 X-v 20 n 2-4835$

João Vitor Roque Guerrero, Daniel Tonelli Caiche, Rubia Gomes Morato, Fernando Shinji Kawakubo, Guilherme Augusto Verola Matavel

comunidades com maior presença de imigrantes, famílias de baixa renda, menores níveis de escolaridade e em bairros com residências com menores áreas e padrões construtivos simplificados.

Uma explicação simples para essa distorção na distribuição de cobertura arbórea é que essas áreas demandam investimento para implantação e adequada manutenção (NELSON et al., 2021). Outra questão está associada a formação de novos bairros por agentes do mercado imobiliário e fragilidades no processo de regulação pública, que dificultam e em até alguns casos inviabiliza a implantação de vegetação arbórea devido à espaços reduzidos e inadequados para suporte de vegetação arbórea de grande porte (CARVALHO, 2016).

Como desdobramento desse contexto, as comunidades mais providas economicamente tipicamente têm maior densidade de árvores, em comparação às comunidades de baixa renda. Mesmo que algumas evidências científicas apontem que esta relação carece de maiores estudos, as cidades apresentam um crescente corpo de pesquisas que corrobora a existência de uma tendência da influência de fatores socioeconômicos nas disparidades no acesso a áreas verdes. (CARVALHO, 2016; RILEY; GARDINER, 2020; NELSON et al., 2021;)

Para aprofundar a compreensão das desigualdades sociais na dinâmica espacial de áreas arborizadas, faz-se necessária uma análise qualitativa e abrangente da quantidade de cobertura arbórea associada a variáveis sociodemográficas, como densidade e renda. Tal análise pode dar suporte aos planejadores urbanos, para tomada de decisão e definição de estratégias com foco nas comunidades mais vulneráveis (HAALAND; BOSCH, 2015). No entanto, poucos esforços têm sido direcionados neste problema específico, especialmente em países em desenvolvimento (YOU, 2016). Como as árvores e os benefícios que estas oferecem serão uma ferramenta cada vez mais importante na luta contra os efeitos das mudanças climáticas e na saúde das pessoas, é fundamental ter um entendimento mais preciso da variação espacial destas áreas e os fatores que influenciam nesse cenário (GILL et al., 2007; ELY; PITMAN, 2013; LAFORTEZZA; SANESI, 2020).

Tendo em vista que as comunidades mais vulneráveis são aquelas que mais necessitam dos benefícios decorrentes do sistema de arborização, o entendimento da relação de como árvores são influenciadas espacialmente por fatores socioeconômicos, fornece informações valiosas para garantir que aqueles que estão em maior risco também tenham acesso adequado aos benefícios de amenidades ambientais (NELSON et al., 2021). O objetivo deste artigo é identificar e espacializar a distribuição da cobertura arbórea na cidade de São Carlos/SP e analisar a influência de dados demográficos e socioeconômicos na distribuição equitativa.

\section{METODOLOGIA}

\section{Caracterização da Área de Estudo}

A área de estudo deste trabalho contempla o perímetro urbano de São Carlos, município localizado na região central do estado de São Paulo (Figura 1), que se distribui em uma área total de $1.136,907 \mathrm{~km}^{2}$, com uma população estimada de 254.484 habitantes para o ano de 2020, valores que 
DE QUEM É O VERDE URBANO? UMA ANÁLISE GEOTECNOLÓGICA DA INIQUIDADE ARBÓREA DE SÃO CARLOS, SP

João Vitor Roque Guerrero, Daniel Tonelli Caiche, Rubia Gomes Morato, Fernando Shinji Kawakubo, Guilherme Augusto Verola Matavel

conferem ao município uma densidade demográfica média de aproximadamente 195,15 habitantes por km² (IBGE, 2018).

Figura 1 - Mapa de localização do município de São Carlos

Figure 1 - Location map of the municipality of São Carlos

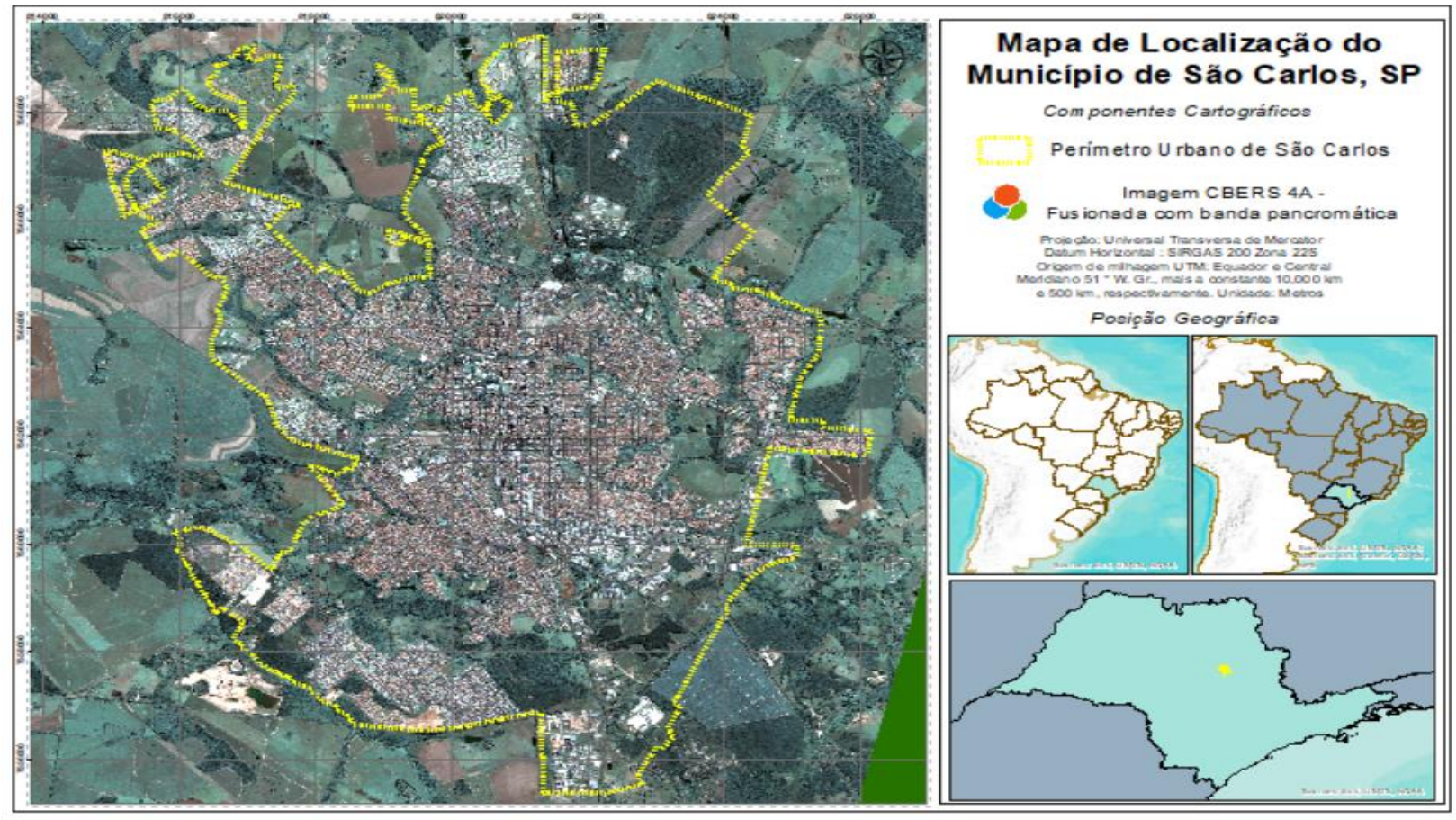

\section{Material e Método}

A abordagem metodológica aplicada nesta pesquisa teve quatro fases principais: 1- Obtenção e processamento dos dados geográficos primários; 2 - Mapeamento da cobertura vegetal arbórea a partir do tratamento e processamento de imagem CBERS 4A; 3 - Geração de índices socioeconômicos de interesse por setores censitários a partir do processamento dos dados do censo 2010 do IBGE e 4 - Mapeamento da Iniquidade Verde do município de São Carlos. A Figura 2 ilustra os procedimentos metodológicos realizados, que são posteriormente descritos. 
DOI: 10.33947/1981-741X-v20n2-4835
DE QUEM É O VERDE URBANO? UMA ANÁLISE GEOTECNOLÓGICA DA INIQUIDADE ARBÓREA DE SÃO CARLOS, SP João Vitor Roque Guerrero, Daniel Tonelli Caiche, Rubia Gomes Morato, Fernando Shinji Kawakubo, Guilherme Augusto Verola Mataveli

Figura 2 - Fluxograma de Métodos

Figure 2 - Methods' flowchart

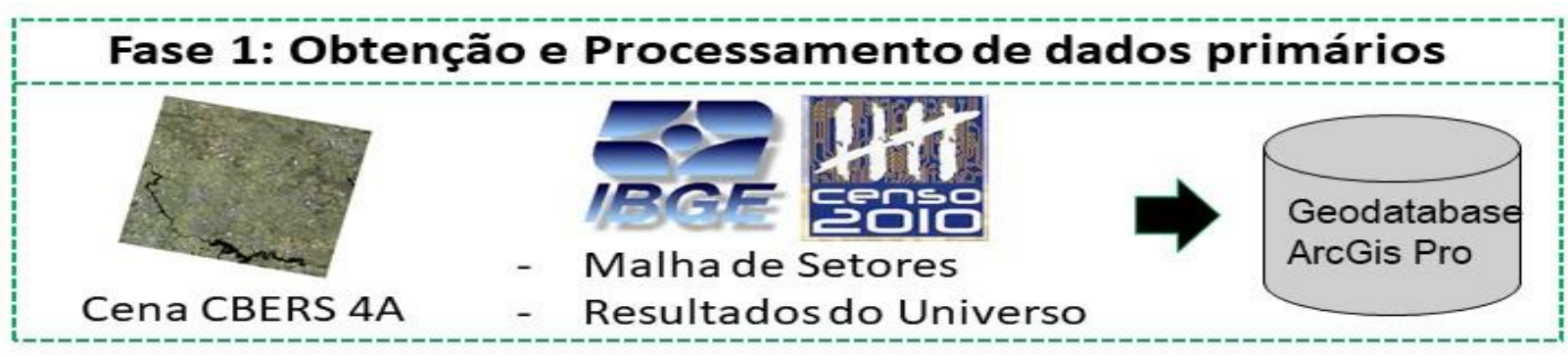

Fase 2: Mapeamento da cobertura vegetal arbórea

- Imagem CBERS 4

Banda Infravermelho

- Imagem CBERS 4

Banda Infravermelho Próximo

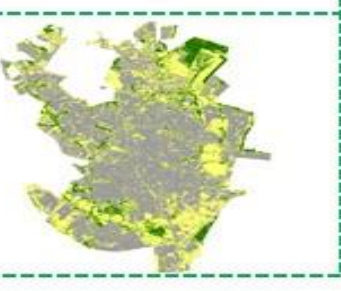

\section{Fase 3: Geração de índices socioeconômicos}

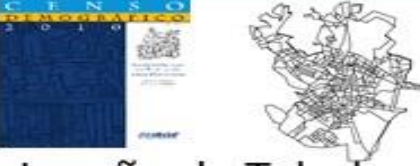

Geração de Índices

Renda média

Junção da Tabela e Densidade Demográfica Malha de Setores

Fase 4: Mapeamento da Iniquidade Verde
$\begin{gathered}\text { Cobertura Vegetal } \\ \text { Arbórea }\end{gathered}$ Socioeconômicos
Indices
Município de São Carlos

Fonte: Elaborado pelos autores (2021). 
DOI: 10.33947/1981-741X-v20n2-4835
DE QUEM É O VERDE URBANO? UMA ANÁLISE GEOTECNOLÓGICA DA INIQUIDADE ARBÓREA DE SÃO CARLOS, SP João Vitor Roque Guerrero, Daniel Tonelli Caiche, Rubia Gomes Morato, Fernando Shinji Kawakubo, Guilherme Augusto Verola Mataveli

\section{Fase 1: Obtenção e Processamento dos dados primários:}

Em prol da quantificação da iniquidade verde do município de São Carlos, foram utilizados dois grupos principais de dados geográficos: Dados geoespaciais provenientes de imagens de satélite e Dados populacionais e territoriais do Censo demográfico brasileiro de 2010 (IBGE, 2010).

O dado de satélite teve como objetivo auxiliar no mapeamento da cobertura vegetal arbórea do município e, para tal, foi utilizada uma cena do satélite Sino Brasileiro CBERS 4A da câmera multiespectral de ampla varredura (WPM), datada de 20/04/2021 com 8 metros de resolução espacial. A aquisição desta cena foi feita de forma gratuita no catálogo de imagens do Instituto Nacional de Pesquisas Espaciais (http://www.dgi.inpe.br/CDSR/), através de uma consulta espacial por área e selecionando as cenas com menos de $5 \%$ de cobertura de nuvens para uma menor interferência das condições atmosféricas na análise final.

Já os dados censitários, provenientes do último censo demográfico do IBGE (2010), contribuíram para a análise da dinâmica socioeconômica de São Carlos e consistiram na malha de setores censitários de 2010 em formato shapefile e dos resultados do universo em formato tabular (.csv), que posteriormente foram agregados à malha em ambiente SIG.

Todos os dados geográficos deste estudo foram padronizados cartograficamente para o sistema de coordenadas planas, projeção Universal Transversa de Mercator (UTM), Datum horizontal SIRGAS 2000, zona 23S.

Por fim, como o interesse deste estudo é estritamente o perímetro urbano, tanto as imagens quanto os setores censitários foram recortados com o limite urbano disponibilizado pelo IBGE (2020).

\section{Fase 2: Mapeamento da cobertura vegetal arbórea}

O mapeamento da cobertura vegetal arbórea do município de São Carlos se deu a partir do processamento digital de imagens (PDI) da cena 20200417_205_141_L4 de 2021 do satélite CBERS 4A, já recortada para o limite do perímetro urbano do município de São Carlos.

O processamento consistiu na aplicação da técnica Normalized Difference Vegetation Index (NDVI), um índice de vegetação que visa identificar o vigor atual das plantas permitindo detectar a presença/ausência de vegetação bem como seu estado a partir da análise normalizada da diferença entre as bandas do vermelho (que absorve fortemente a energia) e infravermelho próximo (que reflete mais os processos vegetativos) (MORENO et al., 2020)

Assim, a aplicação do NDVI se deu a partir da seguinte equação (Equação 1):

$$
\begin{gathered}
\text { NDVI = BV + BIV / BV-BIV } \\
\text { Onde: BV = Banda Vermelho } \\
\text { BVI = Banda Infravermelho Próximo }
\end{gathered}
$$


DOI: 10.33947/1981-741X-v20n2-4835
DE QUEM É O VERDE URBANO? UMA ANÁLISE GEOTECNOLÓGICA DA INIQUIDADE ARBÓREA DE SÃO CARLOS, SP João Vitor Roque Guerrero, Daniel Tonelli Caiche, Rubia Gomes Morato, Fernando Shinji Kawakubo, Guilherme Augusto Verola Matavel

Após a aplicação matemática pixel-a-pixel da equação do NDVI, resultam valores que variam entre -1 e 1 (devido ao caráter normalizador do índice), que representam a condição atual da paisagem quanto à presença ou não de vegetação. A reclassificação dos valores resultantes se deu a partir das classes propostas por Hashim et al. (2019) e Wan \& Gao (2020), apresentadas na tabela 1:

Tabela 1. Valores de NDVI e suas descrições Table 1. NDVI values and their descriptions

\begin{tabular}{|c|c|}
\hline $\begin{array}{c}\text { Valor do } \\
\text { NDVI }\end{array}$ & Descrição \\
\hline & \\
-1 a 0,99 & $\begin{array}{r}\text { Não } \begin{array}{r}\text { Vegetação - Construções, rodovias, áreas } \\
\text { áridas, solo exposto, etc. }\end{array} \\
\hline 0,2 \text { a } 0,5\end{array}$ \\
\hline 0,501 a 1 & Arbustos, gramíneas e pastagens \\
\hline
\end{tabular}

Fonte: Elaborado pelos autores (2021).

\section{Fase 3: Geração de índices socioeconômicos}

A partir do download e importação dos dados tabulares e geográficos do Censo 2010 para o ArcGis Pro, foram gerados os índices socioeconômicos de interesse ao estudo. Inicialmente, foi necessário unir a tabela Básico 1 (que contém dados de população e renda) à malha de setores censitários, com a utilização da ferramenta Join.

Para o mapeamento da densidade populacional, foi utilizada a variável renda proveniente da coluna v002 - que apresenta a quantidade de moradores em domicílios particulares permanentes ou população residente em domicílios particulares permanentes - e a área dos setores censitários a partir da seguinte Equação 2:

Densidade Populacional $=\mathrm{Hab} /$ Area

Onde:

$\mathrm{Hab}=$ Total de habitantes do setor censitário

Area $=$ Área do setor censitário calculada em hectares

Os valores resultantes foram reclassificados em seis classes de acordo com os princípios metodológicos de Nucci (2008).

Já o mapeamento de renda foi produzido a partir da variável V009, que determina o valor do rendimento nominal médio mensal das pessoas de 10 anos ou mais de idade (com e sem rendimento). 


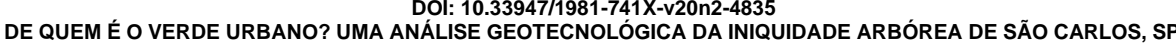
João Vitor Roque Guerrero, Daniel Tonelli Caiche, Rubia Gomes Morato, Fernando Shinji Kawakubo, Guilherme Augusto Verola Mataveli

Os valores de renda foram reclassificados em cinco classes, de acordo com as faixas de classes sociais em relação ao salário-mínimo do IBGE no ano de referência.

\section{Fase 4: Mapeamento da Iniquidade Arbórea do município de São Carlos}

$\mathrm{O}$ conceito de iniquidade remete a injustiças, ou seja, àquilo que é o contrário do justo e igualitário. Assim, a iniquidade verde representa a injustiça da distribuição das áreas verdes (com vegetação arbórea) e de seus serviços ecossistêmicos resultantes de forma igualitária dentro dos espaços urbanos (FERGUSON et al., 2018).

A partir de um robusto e crescente corpo de pesquisas que vêm sendo desenvolvido ao longo das últimas décadas, pode-se concluir o sistema de arborização urbana, ou seja o conjunto de todas as árvores de uma cidade, representa uma infraestrutura fundamental para a vida nas cidades, assim como o sistema de abastecimento de água, coleta de esgoto, transmissão de energia, mobilidade, entre outros (LIOTTA et al., 2020).

Os benefícios ecológicos, sociais e econômicos, influenciam diretamente no cotidiano das pessoas, seja através do amortecimento de águas pluviais e mitigação de enchentes, seja melhorando a qualidade do ar e do microclima, diminuindo taxas de hormônios associados ao stress, ou seja, aumentando a vida útil e diminuindo o tempo de manutenção de outras infraestruturas como o asfalto das vias (DE GROOT et al., 2002).

Para este trabalho, foram levados em consideração para indicar as áreas com maiores iniquidades verdes do município de São Carlos os parâmetros de renda média e densidade populacional, espacializados de acordo com os setores censitários atribuídos pelo IBGE.

Assim, a iniquidade verde foi diagnosticada a partir da análise geoespacial entre a camada que representa as áreas com cobertura vegetal arbórea e as camadas que demonstram os índices socioeconômicos de renda e densidade populacional, apresentando espacialmente as maiores desigualdades para o município de São Carlos.

Finalmente, com o objetivo de identificar espacialmente a relação entre os setores censitários (que promovem a esse estudo o perfil socioeconômico do município) e das áreas arborizadas, foi produzido o mapa de porcentagem de cobertura arbórea de cada setor censitário.

Assim, a iniquidade arbórea do município de São Carlos foi diagnosticada a partir da análise entre os elementos cartográficos produzidos e do conhecimento empírico da dinâmica territorial local.

\section{RESULTADOS E DISCUSSÃO}

\section{Mapeamento da Cobertura Vegetal Arbórea do município de São Carlos}

O mapa de cobertura vegetal arbórea, gerado a partir do índice NDVI estimado pela imagem CBERS 4A, indicou que São Carlos apresenta $9,6 \mathrm{~km}^{2}$ de cobertura arbórea, o que representa $11 \%$ do território (Figura 3). 
DE QUEM É O VERDE URBANO? UMA ANÁl: 10.33947/1981-741X-v20n2-4835 João Vitor Roque Guerrero, Daniel Tonelli Caiche, Rubia Gomes Morato, Fernando Shinji Kawakubo, Guilherme Augusto Verola Matavel

Figura 3 - Mapa de Cobertura Vegetal

Figure 3 - Vegetation Cover Map



Mapa de cobertura vegetal arbórea do município de São Carlos, SP

\begin{tabular}{|c|c|c|c|}
\hline \multicolumn{3}{|c|}{ Classes geradas pelo NDVI } \\
\hline Cor & Valor NDVI & Descrição & Area km² (\%) \\
\hline & -1 a 0,199 & Não Vegetação & $45,9(54 \%)$ \\
\hline & 0,2 a 0,5 & Arbustos e Gramineas & $29,95(35 \%)$ \\
\hline & 0,501 a 1 & Cobertura Arbórea & $9,6(11 \%)$ \\
\hline
\end{tabular}

Convençōes Cartográficas

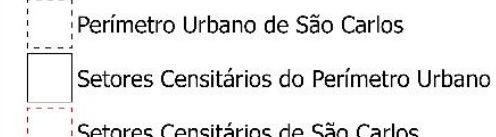

Setores Censitários de São Carlos
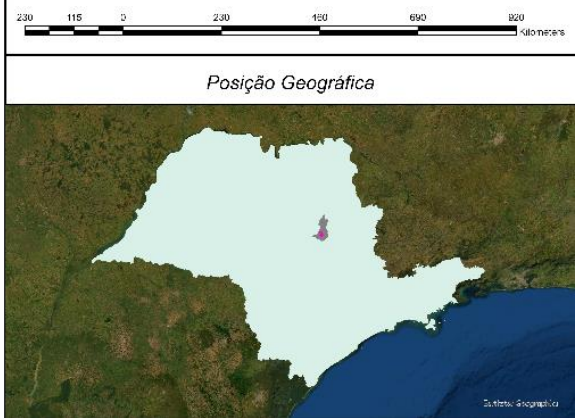

Fonte: Elaborado pelos autores (2021).

A distribuição da cobertura arbórea ocorre de maneira heterogênea ao longo do município, mas é possível notar que as maiores concentrações ocorrem nas áreas periurbanas, principalmente nas áreas norte e sul. Existem ainda duas áreas de especial interesse para a análise da iniquidade arbórea no município de São Carlos que devem ser levadas em consideração por sua dinâmica peculiar quanto à presença arbórea: A Região da Universidade Federal de São Carlos (no norte do município), ilustrada pela Figura 3.1 e o degrau topográfico conhecido como Serra do Cidade Aracy (no sul do município), representada pela Figura 3.2.

A área ao norte da cidade, corresponde ao setor com maior porcentagem de cobertura arbórea. No entanto, é preciso pontuar que parte deste resultado se explica pelo remanescente de plantios comerciais da espécie Pinnus elliotti, cultivado na década de 80 dentro dos limites da Universidade Federal de São Carlos. Esse fragmento florestal tem grande influência nos resultados encontrados.

Já a área ao sul, degrau topográfico da Serra do Aracy, por se apresentar como franja urbana, caracterizada como área rural, nos recentes processos de parcelamento do solo para implantação de 
DOI: 10.33947/1981-741X-v20n2-4835
DE QUEM É O VERDE URBANO? UMA ANÁLISE GEOTECNOLÓGICA DA INIQUIDADE ARBÓREA DE SÃO CARLOS, SP João Vitor Roque Guerrero, Daniel Tonelli Caiche, Rubia Gomes Morato, Fernando Shinji Kawakubo, Guilherme Augusto Verola Mataveli

loteamentos caracterizados por habitação de interesse social, foi requerido nos processos de licenciamento a averbação de fragmentos florestais de fisionomias de cerrado como Reservas Legais. Estas áreas também exercem grande influência na obtenção dos resultados de cobertura arbórea urbana.

É possível afirmar que em ambos os casos, é positiva a presença de fragmentos florestais remanescentes no contexto urbano, quando considerado os benefícios ecológicos como fonte de alimento e abrigo para fauna, produção de oxigênio, regulação do microclima e atenuação das águas pluviais. No entanto, não se trata necessariamente de árvores consideradas típicas de arborização urbana como as árvores de calçadas, canteiros centrais, rotatórias, praças e dentro das edificações, que são árvores projetadas e plantadas para ocupar um espaço específico e que se relacionam com demais elementos de sistemas de infraestrutura urbana.

\section{Índices socioeconômicos do município de São Carlos}

Os índices socioeconômicos de renda média e densidade populacional do município de São Carlos são apresentados na Figura 4. É importante notar que os valores diagnosticados remetem aos dados mais recentes atualmente disponíveis, ou seja, o ano de 2010 , data em que foi realizado o último censo demográfico no Brasil. 
Figura 4 - Mapas de índices Socioeconômicos

Figure 4 - Socioeconomic Index Maps

DENSIDADE POPULACIONAL

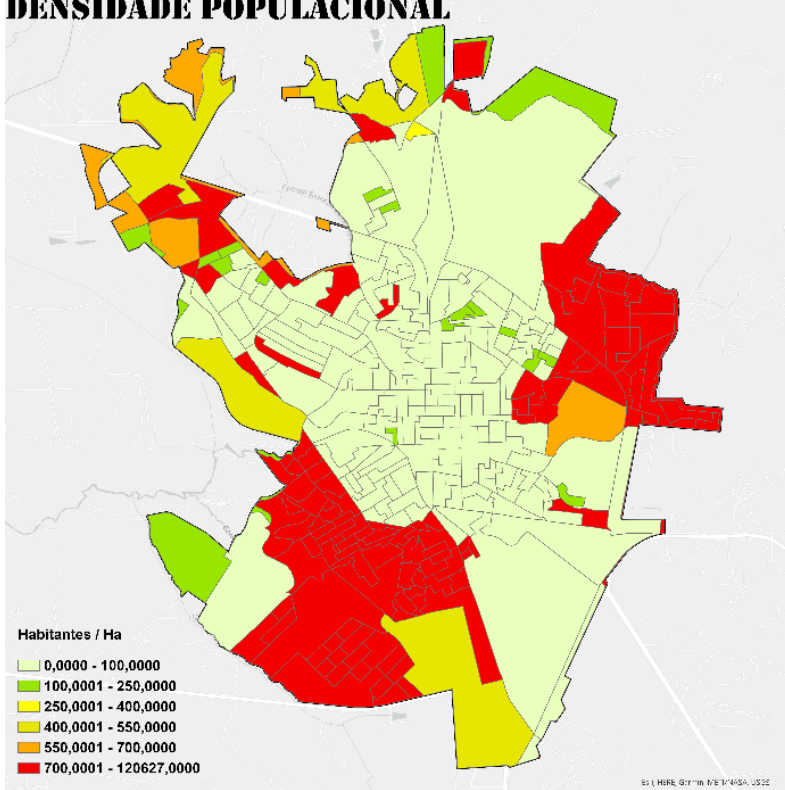

RENDA

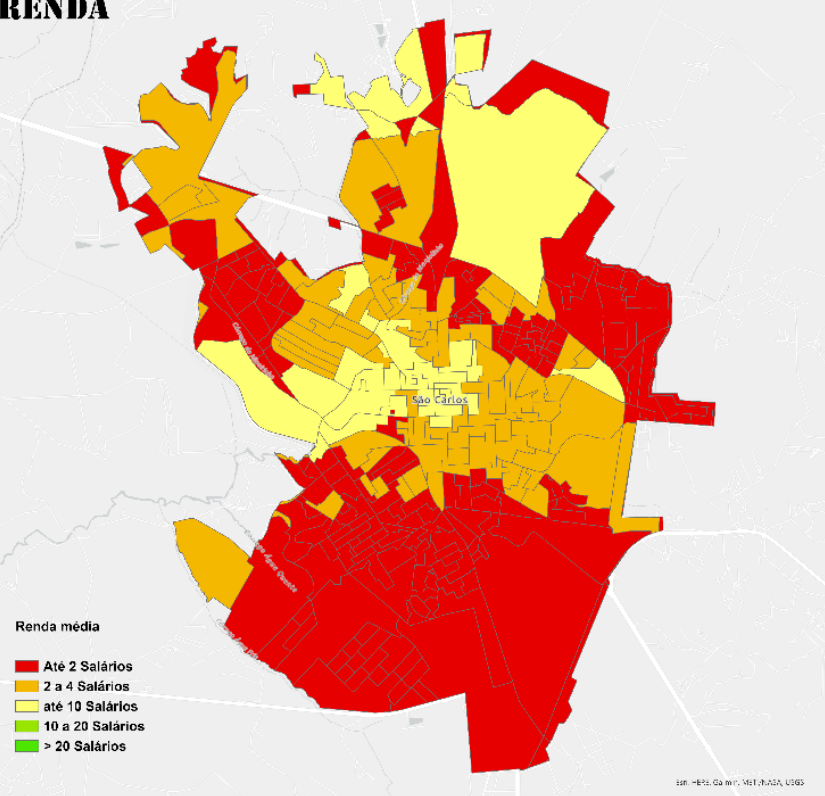

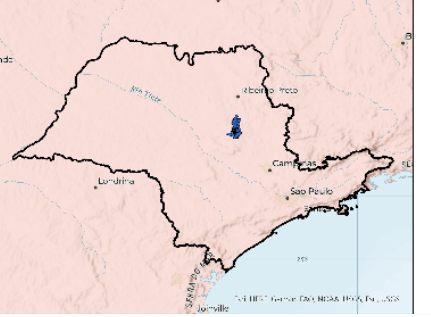

\section{Índices socioeconômicos do município de} São Carlos: Densidade Populacional e Renda

Fonte de dados: Malha de Setores Censitários e Dados Resultados do Universo do Censo 2010 do IBGE para o municipio de São Carlos, SP Disponivel em: www.ibge.gov.br

Produção: Autores (2021)

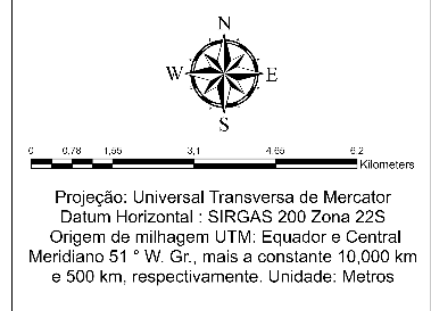

Fonte: Elaborado pelos autores (2021).

Para a densidade populacional, nota-se que há um padrão de concentração de valores mais altos nas regiões sul e nordeste do município, contemplando bairros como os loteamentos de interesse social Cidade Aracy I e II, Jardim Social Antenor Garcia, Jardim Social Presidente Collor e Residencial Itamaraty, todos sendo loteamentos com valores venais mais baixos em relação aos demais bairros de São Carlos e com maior adensamento construtivo e populacional.

É possível identificar também que as duas porções de baixa densidade localizadas na área sul de São Carlos não correspondem a bairros urbanizados atualmente, o que resulta em baixas densidades mesmo em uma região com maior adensamento.

Já para a renda média nos setores censitários foi notado que as maiores concentrações de renda estão localizadas na porção centro-norte do município. Além da região central que configura acesso à melhores redes de transporte e comércio, tal configuração também é reflexo de uma exploração territorial por parte da especulação imobiliária que acaba afastando as populações mais pobres para as áreas com menor valorização do terreno e, consequentemente, com acesso mais dificultado aos equipamentos urbanos essenciais como redes de transporte, saúde e comércio. 
DOI: 10.33947/1981-741X-v20n2-4835

João Vitor Roque Guerrero, Daniel Tonelli Caiche, Rubia Gomes Morato, Fernando Shinji Kawakubo, Guilherme Augusto Verola Mataveli

É possível destacar que as áreas de até 10 salários identificadas nas porções norte e oeste do município sofrem influência espacial das duas maiores Universidades locais (USP e UFSCar), que se tornam grandes polos atrativos de renda por meio de comércios, aluguéis e outras atividades relacionadas. Já as rendas mais elevadas no extremo norte são provenientes de condomínios de alto padrão ali localizados, como os diversos empreendimentos da incorporadora Dhama.

O mapeamento também identificou possíveis efeitos da falta de dados censitários atuais, perceptíveis pela discrepância entre os valores obtidos pelo IBGE no Censo 2010 e a realidade local, como por exemplo as áreas mais ao norte classificadas como de renda de até dois salários não serem compatíveis com a atual ocupação local: Condomínios de alto padrão aquisitivo com os valores venais mais altos do município. O Censo previsto para 2020 não foi realizado e o uso de dados desatualizados traz limitações para inúmeras pesquisas. Assim, é imperativo analisar o território de maneira integrada, a partir de diferentes fatores e principalmente do conhecimento empírico da área de estudo.

Quando analisados os dois mapas em conjunto, é possível observar uma correlação espacial entre densidade populacional alta e renda média baixa nas regiões sul e nordeste do município seguindo o padrão das cidades médias citado por Miguel e Ortigoza (2009), onde as camadas mais pobres da população das cidades médias acomodam-se em áreas periféricas e carentes de serviços urbanos. Por outro lado, observamos que as maiores rendas médias estão relacionadas a áreas menos povoadas de São Carlos, especificamente territórios incorporados por condomínios de luxo.

Outra dinâmica socioespacial que deve ser mencionada para o município de São Carlos são as porções territoriais com maior renda e menor densidade localizadas nas regiões norte e noroeste do município. Estas regiões são habitadas essencialmente por estudantes da Universidade de São Paulo e Universidade Federal de São Carlos, que acabam não contando como residentes de São Carlos, mas que alimentam o comércio e serviços da região via consumo de bens.

\section{Iniquidade arbórea do município de São Carlos}

Ao analisarmos a relação entre as áreas verdes e os índices socioeconômicos o mapeamento indica que a renda média dos locais com a presença de cobertura vegetal arbórea é de $R \$ 1225$ (na classe de 2 a 4 salários-mínimos para o ano de referência), valor ligeiramente superior à média para todo o perímetro urbano ( $\mathrm{R} \$ 1160)$. Já a densidade populacional média para as áreas com cobertura arbórea atinge o valor de 2000 hab./ha, reflexo de uma alta densidade para todo o perímetro urbano de São Carlos (IBGE, 2020).

A distribuição da cobertura arbórea no município de São Carlos por meio do Mapa de porcentagem de cobertura arbórea de cada setor censitário (Figura 5) permitiu notar a dinâmica espacial da arborização local, que concentra as menores taxas de arborização da região central e mais urbanizada e as maiores taxas de arborização nas regiões mais afastadas e menos urbanizadas. 
DE QUEM É O VERDE URBANO? UMA ANÁLISE GEOTECNOLÓGICA DA INIQUIDADE ARBÓREA DE SÃO CARLOS, SP João Vitor Roque Guerrero, Daniel Tonelli Caiche, Rubia Gomes Morato, Fernando Shinji Kawakubo, Guilherme Augusto Verola Mataveli

Figura 5 - Mapa de porcentagem de cobertura arbórea de cada setor censitário Figure 5 - Tree cover percentage map for each census sector

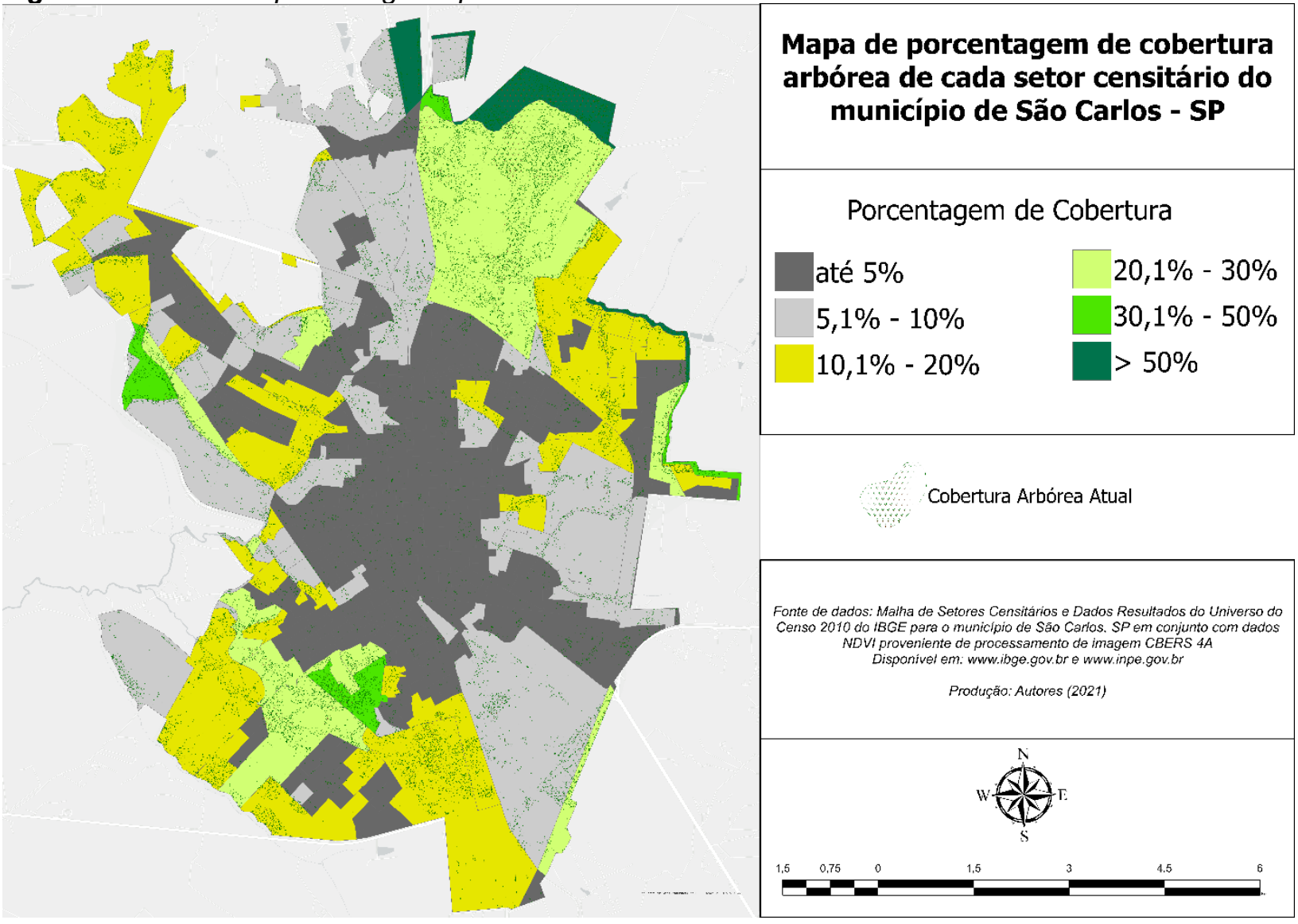

Fonte: Elaborado pelos autores (2021).

As áreas com maior cobertura arbórea percentual (> 50\%) estão no extremo norte da cidade, em regiões periurbanas, transitórias entre o urbano e o rural. Estas áreas representam características específicas: a área protegida de cerrado da Universidade Federal de São Carlos e as áreas verdes particulares pertencentes aos condomínios de luxo ali instalados. De acordo com Lima et al. (2020), as maiores taxas de arborização urbanas concentradas em condomínios de luxo evidenciam um processo de segregação socioeconômica sobretudo em relação ao direito à cidade arborizada, onde concentra-se o "verde" urbano em empreendimentos privados, privando a população menos abastada dos serviços ecossistêmicos ali prestados.

Considerando que estudos recentes na temática de arborização urbana, tais como Grove et al. (2006) e Kondo et al. (2020), indicam 30\% como sendo o valor ideal de cobertura arbórea para os municípios serem mais resilientes, São Carlos ainda tem uma árdua tarefa, já que como apresentado anteriormente, o perímetro urbano contém $11 \%$ de cobertura. Esses dados são ainda agravados quando o foco é nos setores censitários, já que apenas 2\% (7 setores) dos setores são carlenses apresentam cobertura arbórea maior do que 30\%. Dentre esses 7 setores, apenas 3 efetivamente 
representam territórios urbanizados (Residencial Dhama, Bosque São Carlos e Cidade Aracy), sendo que os demais representam os campi universitários ou remanescentes florestais em áreas não loteadas.

Para as áreas de 10 a 20\% cobertura mapeadas, emergem diversas preocupações socioambientais. A primeira é que esses locais (identificados em amarelo na Figura 5) contém altas densidades demográficas e baixas rendas per capita, caracterizando-se por bairros periféricos e carentes, sugerindo uma segregação ambiental já que essas populações pobres têm pouco acesso aos serviços ecossistêmicos prestados pela arborização urbana.

Além disso, a baixa cobertura vegetal combinada com um meio físico vulnerável desses locais indica também elevados potenciais de ocorrência de problemas ambientais, tais como erosão e movimentos de massa (MASSA; ROSS, 2012; ROSS, 1994). Essa afirmação é suportada pelos resultados encontrados por Rissi et al. (2021), que indicam que a região sul de São Carlos apresenta fragilidade ambiental muito forte com potenciais riscos também aos moradores locais.

Já os setores com menos de $10 \%$ de cobertura arbórea estão localizados principalmente nas porções central e norte, sendo que do total de setores, 83 \% estão nessa situação. Esses resultados demonstram que o município ainda carece de esforços para multiplicar a cobertura arbórea nas áreas mais urbanizadas do município, propagando, assim, os diversos serviços ecossistêmicos prestados pelas áreas verdes, tanto nos aspectos ecológicos, quanto na prestação de serviços ecossistêmicos culturais, assim como propõe De Groot et al. (2010).

Uma preocupação adicional provém dos setores mais ao sul, que também de acordo com Rissi et al. (2021), encontram-se em áreas de forte fragilidade ambiental, agravada pela baixa taxa de cobertura arbórea e que concentram altas densidades demográficas de uma população também vulnerável pela baixa renda local.

\section{CONCLUSÃO}

O mapeamento da iniquidade arbórea demonstrou uma complexa dinâmica territorial para o município de São Carlos, mas averiguou que as maiores zonas da cobertura arbórea municipal tendem a pertencer a três grupos principais: Condomínios fechados de alto poder aquisitivo, Campi universitários e remanescentes florestais em áreas não loteadas.

Apesar de notarmos que o município ainda apresenta grandes iniquidades arbóreas em relação ao acesso da população mais carente a esse recurso, é notável que o município ainda contém remanescentes florestais importantes, provenientes de áreas de conservação que prestam serviços ecossistêmicos importantes, inclusive próximo a áreas carentes. Cabe ao poder público transformar esses espaços em áreas de lazer e cultura para a população local, aumentando a prestação de serviços ecossistêmicos culturais.

Um problema sério, enfrentado atualmente por diversas pesquisas que utilizam dados socioeconômicos é o atraso na realização do censo demográfico previsto para 2020, adiado para 
DE QUEM É O VERDE URBANO? UMA ANÁLISE GEOTECNOLÓGICA DA INIQUIDADE ARBÓREA DE SÃO CARLOS, SP João Vitor Roque Guerrero, Daniel Tonelli Caiche, Rubia Gomes Morato, Fernando Shinji Kawakubo, Guilherme Augusto Verola Matavel

2021 e depois para 2022. Em estudos urbanos, transformações consideráveis podem acontecer em períodos com mais de 10 anos. Assim, a falta de dados atualizados traz limitações em diversos tipos de pesquisa. Foi o que ocorreu ao norte da área de estudo, onde houve o crescimento recente de condomínios de alto padrão, que foram negligenciados nos mapas e análises de renda devido à desatualização dos dados oficiais.

A partir dos resultados encontrados neste estudo e, para que possamos seguir no caminho de atingir as diretrizes previstas na Lei Federal o 10.257 de 2001 (Estatuto da Cidade), mais especificamente no que diz respeito à "garantia do direito a cidades sustentáveis" e a "justa distribuição dos benefícios e ônus decorrentes do processo de urbanização", é recomendável que o poder público proponha ações de arborização dentro do contexto do desenvolvimento urbano a partir de estudos técnicos que o orientem.

Assim, espera-se que os resultados aqui obtidos possam de alguma forma subsidiar o poder público em suas tomadas de decisão acerca dos projetos de arborização urbana, além de incentivar a arborização urbana como instrumento de desenvolvimento socioambiental dos municípios brasileiros.

\section{REFERÊNCIAS BIBLIOGRÁFICAS}

CARVALHO, R. M. R. O discurso ambientalista e a mercadoria da paisagem: papéis dialéticos assumidos pelas áreas naturais no processo de organização espacial da cidade capitalista. InSitu, v. 2, n. 2, p. 3-26, 2016.

\section{DAVERN, M. et al. Quality Green Space Supporting Health, Wellbeing and Biodiversity: A} Literature Review. Melbourne: [s.n.], 2017.

DE GROOT, R. S.; WILSON, M. A; BOUMANS, R. M. J. A typology for the classification, description and valuation of ecosystem functions, goods and services. Ecological Economics, v. 41, n. May, p. 1-20, 2002.

DE GROOT, R. S. et al. Challenges in integrating the concept of ecosystem services and values in landscape planning, management and decision making. Ecological Complexity, v. 7, n. 3, p. 260272, 2010. Disponível em: <http://dx.doi.org/10.1016/j.ecocom.2009.10.006>.

DONOVAN, G. H.; PRESTEMON, J. P. The effect of trees on crime in Portland, Oregon. Environment and Behavior, v. 44, n. 1, p. 3-30, 2012.

ELY, M.; PITMAN, S. Green Infrastructure: Life support for human habitats. A review of research and literature: Prepared for the Green Infrastructure Project. Botanic Gradens of Adelaide, Department of Environment, Water, and Natural Resoruces. p.340, 2013.

FERGUSON, M. et al. Contrasting distributions of urban green infrastructure across social and ethno-racial groups. Landscape and Urban Planning, v. 175, n. March, p. 136-148, 2018.

GILL, S. E. et al. Adapting Cities for Climate Change: The Role of the Green Infrastructure. Built Environment, v. 33, n. 1, p. 115-133, 2007. 
GILSTAD-HAYDEN, K. et al. Research note: Greater tree canopy cover is associated with lower rates of both violent and property crime in New Haven, CT. Landscape and Urban Planning, v. 143, p. 248-253, 2015.

GROVE, J. M. et al. A report on New York City's present and possible urban tree canopy, prepared for: Fiona Watt, Chief of the Division of Forestry and Horticulture. New York Department of Parks and Recreation, USDA Forest Service, Northern Research Station. p. 28, 2006.

HAALAND, C.; BOSCH; C. K. V. D. Challenges and strategies for urban green-space planning in cities undergoing densification: A review. Urban Forestry \& Urban Greening, v. 14, n. 4, p. 760$771,2015$.

HASHIM, H.; ABD LATIF, Z.; ADNAN, N. A. Urban vegetation classification with NDVI threshold value method with very high resolution (VHR) pleiades imagery. International Archives of the Photogrammetry, Remote Sensing and Spatial Information Sciences - ISPRS Archives, v. 42, n. 4/W16, p. 237-240, 2019.

IBGE. Censo Demográfico (2010). Disponível em:http://www. censo2010. ibge. gov. br/. Acesso em: 23 ago. 2021.

IBGE Cidades-Brotas, SP. Disponível em:

https://cidades.ibge.gov.br/brasil/sp/brotas/panorama. Acesso em: 22 maio 2018.

. Mapas IBGE (2020). Disponível em:

https://portaldemapas.ibge.gov.br/portal.php\#homepage. Acesso em: 15 ago. 2021.

KIMPTON, A. A spatial analytic approach for classifying greenspace and comparing greenspace social equity. Applied Geography, v. 82, p. 129 - 142, 2017.

KONDO, M. C. et al. Health impact assessment of Philadelphia's 2025 tree canopy cover goals. The Lancet Planetary Health, v. 4, n. 4, p. e149 - e157, 2020. Disponível em: http://dx.doi.org/10.1016/S2542-5196(20)30058-9.

KUEHLER, E.; HATHAWAY, J.; TIRPAK, A. Quantifying the benefits of urban forest systems as a component of the green infrastructure stormwater treatment network. Ecohydrology, n. 10: e1813, p. 1-10, 2017.

LAFORTEZZA, R.; SANESI, G. Nature-based solutions: Settling the issue of sustainable urbanization. Environmental Research, v. 172, p. 394 - 398, 2020.

LIMA, G. V. B. A. et al. O direito à cidade arborizada: a arborização urbana como indicador da segregação socioeconômica em Belém do Pará. Revista da Sociedade Brasileira de Arborização Urbana, v. 15, n. 1, p. 79, 2020.

LIOTTA, C. et al. Planning for environmental justice - reducing well-being inequalities through urban greening. Environmental Science and Policy, v. 112, n. June, p. 47-60, 2020.

LIVESLEY, S. J.; MCPHERSON, G. M.; CALFAPIETRA, C. The urban forest and ecosystem services: Impacts on urban water, heat, and pollution cycles at the tree, street, and city scale. Journal of Environmental Quality, v. 45, n. 1, p. 119-124, 2016.

MASSA, E. M.; ROSS, J. L. S. Aplicação de um modelo de fragilidade ambiental relevo-solo na Serra da Cantareira, Bacia do Córrego do Bispo, São Paulo-SP. Geography Department, 
University of São Paulo, v. 24, n. 2012, p. 57-79, 2012. Disponível em: http://www.revistas.usp.br/rdg/article/view/52754.

MCCORMICK, R. Does access to green space impact the mental well-being of children: a systematic review. Journal of Pediatric Nursing, v. 37, p. 3-7, 2017.

MIGUEL, Y. D. C.; ORTIGOZA, S. A. G. A segregação sócio-espacial contemporânea Introdução. 2009, Montevideu: Egal - Encuentro de Geógrafos de América Latina, p. 6, 2009.

MORENO, R. et al. Application of NDVI for identify potentiality of the urban forest for the design of a green corridors system in intermediary cities of Latin America: Case study, Temuco, Chile. Urban Forestry and Urban Greening, v. 55, 2020.

NELSON, J. R. et al. The equity of tree distribution in the most ruthlessly hot city in the United States: Phoenix, Arizona. Urban Forestry \& Urban Greening, v. 59, p. 127016, 2021.

NESBITT, L. et al. Who has access to urban vegetation? A spatial analysis of distributional green equity in 10 US cities. Landscape and Urban Planning, v. 181, p. 51-79, 2019.

NUCCI, J. C. Qualidade ambiental e adensamento urbano. [S.I: s.n.], 2008. Disponível em: http://www.geografia.ufpr.br/laboratorios/labs.

RILEY, B. C.; GARDINER, M. M. Examining the distributional equity of urban tree canopy cover and ecosystem services across United States cities. Plos One, p. 1-22, 2020.

RISSI, P. R. et al. Analysis of zoning of the master plan and its relation with structural landscape components of the municipality of São Carlos - SP. Anuario do Instituto de Geociencias, v. 44, n. 1, p. 1-12, 2021.

ROSS, J. L. S. Análise empírica da fragilidade dos ambientes naturais e antropizados. Revista do Departamento de Geografia, São Paulo, 1994.

RUGEL, E. J. et al. Exposure to natural space, sense of community belonging, and adverse mental health outcomes across an urban region. Environmental Research, v. 171, p. 365-377, 2019.

SCHWARZ, K. et al. Trees grow on Money: urban tree canopy cover and environmental justice. Plos One, p. 1-17, 2015.

SHACKLETON, S. et al. Multiple benefits and values of trees in urban landscapes in two towns in northern South Africa. Landscape and Urban Planning, v. 136, n. 295, p. 76-86, 2015.

TYRVÄINEN, L. et al. The influence of urban green environments on stress relief measures: A field experiment. Journal of Environmental Psychology, v. 38, p. 1-9, 2014.

TZOULAS, K. et al. Promoting ecosystem and human health in urban areas using Green Infrastructure: A literature review. Landscape and Urban Planning, v. 81, n. 3, p. 167-178, 2007.

ULMER, J. M. et al. Health \& Place Multiple health benefits of urban tree canopy: The mounting evidence for a green prescription. Health \& Place journal, v. 42, p. 54-62, 2016.

WAN, Z.; GAO, W. Changes in urban vegetation cover and analysis of the influencing factors: a case study of Harbin, Heilongjiang Province, China. Arabian Journal of Geosciences, v. 13, n. 19, 2020. 
DOI: 10.33947/1981-741X-v20n2-4835
DE QUEM É O VERDE URBANO? UMA ANÁLISE GEOTECNOLÓGICA DA INIQUIDADE ARBÓREA DE SÃO CARLOS, SP João Vitor Roque Guerrero, Daniel Tonelli Caiche, Rubia Gomes Morato, Fernando Shinji Kawakubo, Guilherme Augusto Verola Matavel

WARD THOMPSON, C. et al. More green space is linked to less stress in deprived communities: Evidence from salivary cortisol patterns. Landscape and Urban Planning, v. 105, n. 3, p. 221-229, 2012.

WATKINS, S. L. et al. Does collaborative tree planting between nonprofits and neighborhood groups improve neighborhood community capacity? Cities, v. 74, n. November 2017, p. 83-99, 2018.

WERBIN, Z. R. et al. A tree-planting decision support tool for urban heat mitigation. Plos One, v. 15, n. 10 , p. $1-13,2020$.

YOU, H. Characterizing the inequalities in urban public green space provision in Shenzhen, China. Habitat International, v. 56, p. 176-180, 2016.

ZHAO, J. et al. Science of the Total Environment Temporal trend of green space coverage in China and its relationship with urbanization over the last two decades. Science of the Total Environment, v. 442, p. 455-465, 2013. 